\title{
Prostatic paracoccidioidomycosis: differential diagnosis of prostate cancer
}

\author{
Daniel Lima Lopes ${ }^{1}$, Stanley de Almeida Araújo ${ }^{2 /+}$, João Paulo Lemos da Silveira Santos², \\ Ana Claudia Lyon², Diogo Vieira Dantas², Bernardo Sgarbi Reis³, Alfredo Miranda de Góes³, \\ Ênio Roberto Pietra Pedroso ${ }^{2}$
}

\begin{abstract}
${ }^{1}$ Hospital do Instituto de Previdência dos Servidores do Estado de Minas Gerais, Belo Horizonte, MG, Brasil ${ }^{3}$ Departamento de Bioquímica e Imunologia, Instituto de Ciências Biológicas Faculdade de Medicina, Universidade Federal de Minas Gerais, Belo Horizonte, MG, Brasil
\end{abstract}

Symptomatic prostatic paracoccidioidomycosis (PCM) is a very rare condition; however, it may express as a typical benign prostatic hyperplasia or a simulating prostatic adenocarcinoma. This case report presents PCM mimicking prostatic adenocarcinoma. The purpose of this paper is to call the general physician's attention to this important differential diagnosis.

Key words: Paracoccidioides brasiliensis - paracoccidioidomycosis - prostatitis - prostate cancer

Paracoccidioidomycosis (PCM) is an important deep mycosis in Latin America. It is caused by the dimorphic fungus Paracoccidioides brasiliensis, whose spores enter the body via the respiratory tract and evolve either asymptomatically or in a sub-acute or chronic manner. The parasite is able to provoke several clinical presentations due to its capacity to disseminate itself from the lungs through the lymphatic system or via the blood stream to any organ or system. PCM mainly affects adult males, preferentially in the lungs, mucosa, skin and phagocytic-monocytic system (Paniago et al. 2003, Prado et al. 2005). On a smaller scale, it reaches the nervous, musculoskeletal and suprarenal systems (Paniago et al. 2003). Its clinical incidence in the urinary tract, especially in prostatic injuries, is not well known. In a few necropsy studies involving disseminated PCM cases, its incidence varies from 2.7-9\% (Salfelder et al. 1969, Begliomini et al. 1993).

Prostatic diseases have become more common due to the increase in the population's age. Generally, they appear in males over 40-years-old, being more often bacterial and viral infections, benign hyperplasia and adenocarcinoma. Prostatic adenocarcinoma is the most fearful lesion due to its malignant and metastatic potential, and it is seen clinically in $10 \%$ of cases. Its diagnosis must always be set apart because its mortality is $3 \%$, making it the greatest cause of morbi-mortality among men (Crawford 2003).

Fungal prostatitis is unusual, having as its most common agents Coccidioides immitis, Candida albicans, Aspergillus sp., Cryptococcus neoformans and Blastomyces dermatitidis, which mainly affect patients who are immune-suppressed. The symptomatic attack of the prostate by PCM has rarely been described. Its potential severity justifies the reporting of this case.

+ Corresponding autor: stanleyaa@gmail.com

Received 3 September 2008

Accepted 21 January 2009
In this paper, infection of the prostate by $P$. brasiliensis is described, mimicking cancer with urethral obstruction and urgent expansion of the bladder.

\section{Case study}

AAF, a 54-year-old married rural worker who was born in and is a current resident of São Sebastião do Maranhão, Minas Gerais, Brazil, presented in December 2003 with palate stomatitis associated with dysphagia and odynophagia. A biopsy of this injury revealed $P$. brasiliensis. He was treated with Sulfametoxazol (800 $\mathrm{mg}$ ) and Trimethoprim (160 $\mathrm{mg})$ daily in an irregular way for 20 months. In December 2004, he started to complain of dysuria, polyuria and urinary urgency and frequency, which culminated after one year in prompt urinary retention. Concomitantly, a stomatitis with moriforme injury of the palate appeared.

A digital rectal exam revealed an increased prostate volume, induration, precise limits and a nodule on the right lobe. It was thought to be prostate cancer. His PSA level was measured at $0.563 \mathrm{ng} / \mathrm{mL}$ and transrectal ultrasonography demonstrated a prostrate with irregular contours, with peripheral zones containing hypoechoic areas with heterogenic texture (Fig. 1). There was a volumetric increasing of the gland, which weighed $57.9 \mathrm{~g}$. The prostatic biopsy revealed a diffuse chronic granulomatous inflammatory process showing epithelial and multinucleated giant cells, neutrophils and eosinophils involving the $P$. brasiliensis (Fig. 2 A, B).

A complete blood count showed a total counting of leukocytes of $12,940 / \mathrm{mm}^{3}$ (neutrophils $9,614 / \mathrm{mm}^{3}$, lymphocyte $2,057 / \mathrm{mm}^{3}$, eosinophils $349 / \mathrm{mm}^{3}$, basophils $116 /$ $\mathrm{mm}^{3}$, monocytes $660 / \mathrm{mm}^{3}$ ). The urine exam revealed hemoglobin $+/ 4+$, urobilinogen $0.2 \mathrm{mg} / \mathrm{dL}$, leukocytes $+/ 4+$, piocytes $20 /$ field, red blood cells $10 /$ field, bacterian microbiota $+++/ 4+$, rare grouped piocytes and the presence of renal epithelium. Gram staining did not reveal any bacteria and serum biochemistry did not show any abnormalities. A chest X-ray showed localized micro-re- 
ticulo-nodule alterations, mainly on the hilum and in the pulmonar basis bilaterally. Abdominal ultrasonography did not show any alteration and the other urinary organs were apparently normal.

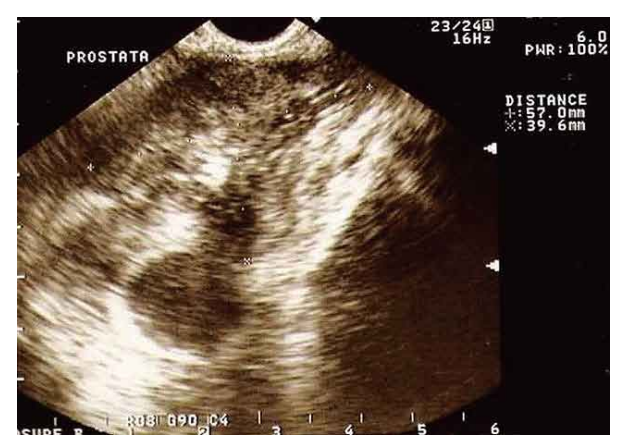

Fig. 1: prostatic transrectal ultra-sound. Prostate with regular contours and precise borders. The peripheric zones present hypoechoic areas with heterogenic textures. Prostrate mesures: longitudinal: $57.0 \mathrm{~mm}$; anti-posterior: $39.6 \mathrm{~mm}$; transverse: $46.6 \mathrm{~mm}$; prostatic weigh: $57.9 \mathrm{~g}$. Data compatible with the general volumetric increasing of the prostate.

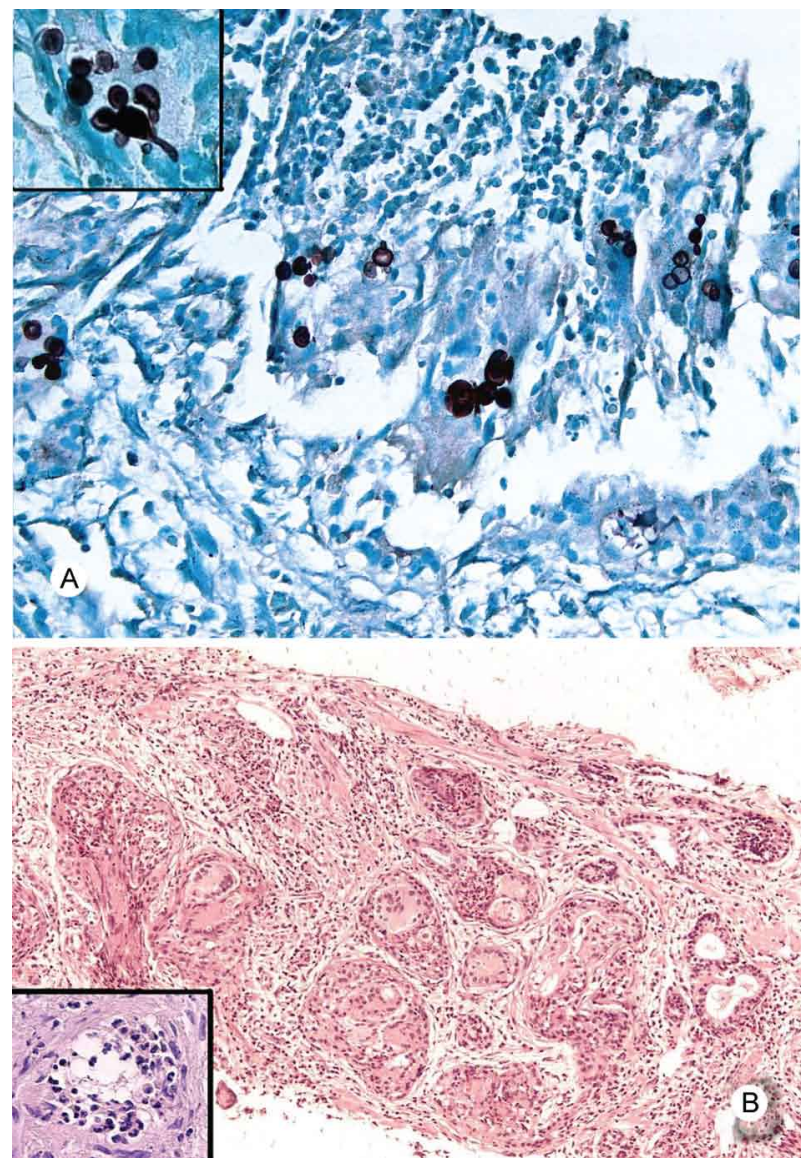

Fig. 2: biopsy by thin needle of prostatic tissue. A (Grocott): many yeasts in the tissue. In detail, yeast with multiple sprouting (400X): $\mathrm{B}$ (HE): chronical granulomatosis inflamatory process, epitheliodcells and multinucleus giant cells involving parasites (100X).
Collected blood samples were positive in anti-P. brasiliensis serologic tests using the antigens PB28, Pbgp43 and membrane and extracellular antigens (MEXO) (Fig. 3).

The initial treatment was done with Itraconazol $(100$ mg per day), which was replaced after 10 days with Sulfametoxazol $(800 \mathrm{mg})$ and Trimetoprim $(160 \mathrm{mg}$ every $12 \mathrm{~h}$ ) because the former was not available at public health care centres in Brazil. After six months, the patient was asymptomatic and without deficits or clinical or radiological residual injuries. The total PSA level remained low despite the intense inflammation (Fig. 4).

After one year of ambulatory observation, there was no recurrence of the infection or side effects from the treatment.

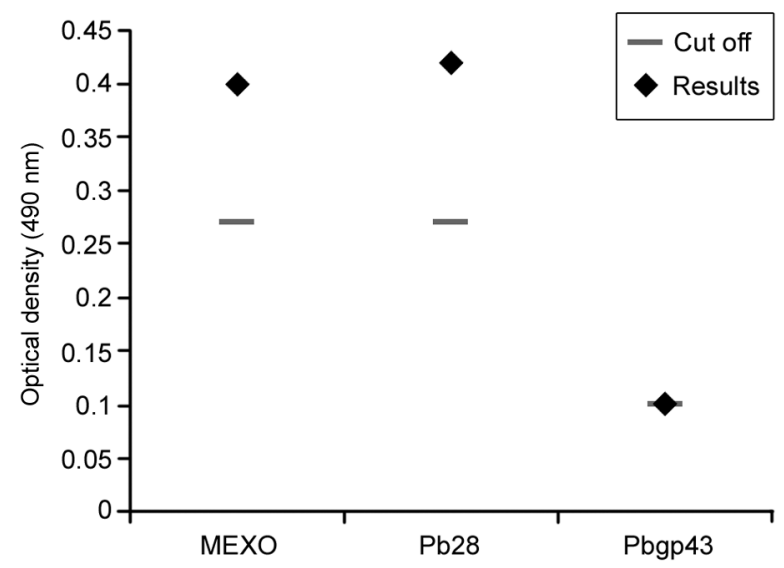

Fig. 3: serum tested by enzyme-linked immunosorbent assays results for the extracelular antigens and membranes, PB28 and Pbgp43, made in the acute fase of patient's disease.

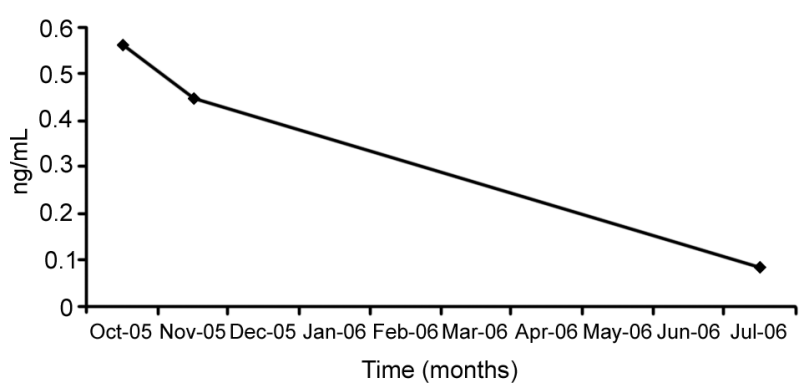

Fig. 4: total prostatic-specific antigen (PSA) serum variation after one year of medical following.

\section{DISCUSSION}

We report this case due to the rare presentation of PCM in the prostate, which may be a serious symptomatic condition if untreated and due to the importance of the differential diagnosis with prostate adenocarcinoma, which is a very severe disease, with lethality and me- 
tastatic potential whose possibility of treatment is only surgical. Depending on the extension of the neoplastic tissue and on the chosen technique, prostatectomy may lead to minor or serious complications such as erectile problems urinary incontinence and damage to the urethra or rectum (Wilt \& Brawer 1999). Thus, a proper histopathological exam may demonstrate an infectious prostatitis and avoid an unnecessary surgical intervention and its related risks.

PCM has special importance in Latin America, where it is endemic among infecto-parasitarian diseases due to the severity and the peculiarity of some of its clinical forms. This infection is not commonly isolated in the urogenital tract. PCM in the prostate is not considered a primary disease, affecting only $1.59 \%$ of patients in the disseminated form (Brito \& Caprini 1959, Ciconelli et al. 1969, Begliomini et al. 1993). All of the urogenital tract may be affected by the disease, including the epididymis, testicles, prostate, ureters, penis, urethra and kidneys. The first case of prostatic involvement was described by Brito and Caprini (1959); since then, only a few other cases have been described (Melo et al. 1992, Severo et al. 2000).

Because of the general symptoms, the diagnosis of PCM is frequently done later than expected. Patients with prostatic involvement may present obstruction symptoms of the lower urinary tract.

Cancer and chronic inflammation always constitute the differential diagnosis for complaints related to prostatic disease symptoms.

When the prostate is involved in PCM infection, differential diagnosis with cancer is difficult due to the variety of damage and absence of specific signs. The diagnosis of prostatic injuries is suggested by the clinical aspects, but it often requires confirmation by a histopathological exam. A biopsy is mandatory due to the necessity for cancer exclusion (Stillwell et al. 1987). Differential diagnosis for PCM of the prostate is divided into specific, caused by bacteria (Treponema pallidum, Mycobacterium tuberculosis, Brucella sp.), fungi (Candida sp., Aspergillus sp., C. neoformans, Histoplasma capsulatum, Actinomyces sp., B. dermatitidis and $C$. immitis), viruses or parasites, and non-specific, derived from xantogranulomatousis, allergies, post-TURP or post-biopsies.

Granulomatous prostatitis is a rare disease that frequently simulates the development of prostatic carcinoma due to the formation of nodules and the increase of the gland by rectal touch (Stillwell et al. 1987, Begliomini et al. 1993). A histopathological exam of the granulomatous prostatitis, however, showed infiltrated lymphoplasmocyte, pseudotubercules, epithelioidic cells and the absence of signs of atypical cellular proliferation, which characterize the neoplasia. In coccidioidomicosis and North American blastomicosis, the involvement of the genitourinary tract is frequent, being common in prostatic attack in the disseminated form (Brito \& Caprini 1959, Stewart 1964, Eickenberg et al. 1975). There have been a few cases of transmission of $B$. dermatitidis from men with prostatic involvement to their female sexual partner (Faber et al. 1968). PCM has no record of inter-human transmission despite its potential for sexual transfer by contaminated prostatic secretion.

Tuberculosis or other mycotic diseases that affect the genitourinary tract are other important differential diagnoses and the microbiologic study of urine and cultures are fundamental for its discernment (Stillwell et al. 1987). Since P. brasiliensis is not coloured by Gram stain, its diagnosis may not be noticed in routine exams.

Induration of the prostate may persist upon physical examination for years and scarring and fibrosis have been observed in a third of the patients (Paniago et al. 2003). The case described here presented sexual dysfunction after the treatment and the effects tobacco use, aging process and adverse effect of the treatment with Sulfametoxazol-Trimetoprim or Ketoconazole, which were concomitant conditions in this case, could be potential factors, in addition to prostate fibrosis induced by PCM (Brito \& Carpini 1959).

There is no report of alterations in serum levels of the prostatic-specific antigen PSA during the treatment. In granulomatous prostatitis, both increasing and decreasing serum levels of PSA may be observed. A significant reduction in PSA, as in this case, suggests that cytokines derived from the activated macrophages and T-lymphocytes may have a regulatory effect on the cells' secretions and cause the destruction of the prostatic epithelia (Dhundee \& Maciver 1991).

Current serum tests are highly sensitive and specific for diagnosis and useful for the clinical follow-up of people affected with PCM. Normal serum results are extremely rare with evidence of the disease's histopathology.

Serum tested by enzyme-linked immunosorbent assays (ELISA) for the extracellular antigens and membranes - MEXO - from P. brasiliensis presents a specificity of $96.6 \%$ compared to control groups and $81.2 \%$ in relation to groups with diverse infections (Reis et al. 2005). A purified protein with a molecular weight of 28-kDa of MEXO, called $\mathrm{Pb} 28$, is the most specific antigen in the humoral immunological response of PCM. This protein reacts and has a specificity of $100 \%$. It may be used as an alternative antigen to the serum diagnostic method. ELISA, either for MEXO or for $\mathrm{Pb} 28$, is a strong reactor in serum analysis when collected during the acute period of the disease (Fig. 4). However, one glycoprotein with a molecular weight of $43 \mathrm{Kda}$, known as Pbgp43, which is an important extracellular component released by $P$. brasiliensis during its pathogenic phase, is used in the detection of the serum antibodies in patients with PCM and in epidemiologic studies through the intradermal reaction (Kalmar et al. 2004). It presents a sensitivity of $95.1 \%$ and a specificity of $97.5 \%$. In this case, it was reactor-moderated (Fig. 4).

Such serum data is in accordance with those reported by Reis et al. (2005) because $\mathrm{Pb} 28$ has proven to be more sensitive and specific for PCM and Pbgp43 presented irregular results.

PCM is diagnosed by the identification of the yeasts in direct exams of clinical samples (biopsy, mucous, scratched) or cultures. The yeast has specific microscopic aspects, including a bi-refringent wall, reproduction by 
multiple-sprouting and originating blastospores that do not detach from the mother cell, which gives them a unique appearance among the pathogenic fungi (Fig. 2A).

The granuloma is formed with the objective of restricting the parasite and avoiding its dissemination (Fig. 2B). Thus, the more efficient the cellular immunologic response is the better the efficiency of the granulomatos reaction and the lesser the severity of the disease. The histopathological response of the host caused by $P$. brasiliensis resembles tissue invasion by $B$. dermatitidis and $C$. immitis.

Injuries of the oropharyngeal mucosa are frequently observed. The most characteristic aspect is moriform ulcerous stomatitis.

PCM is a disease that lacks standards for cure and a standard treatment protocol. There are no specific treatments for the urogenital form. Prolonged medication is, in many cases, necessary to prevent recurrence, which exhausts the patient and impairs his/her adequate compliance with the proposed treatment. Interruption of the medication or irregular or inadequate use may drive the emergence of resistance, making the problem worse and producing several side-effects (Prado et al. 2005).

Once a diagnosis of prostatic PCM is made, a specialist in infectious diseases should be consulted. Finally, the patient needs to be observed for many years for prevention and occasional treatment of disease recurrence.

PCM is a systemic disease with the potential for involvement of all organs and it is necessary to pay attention to all complaints presented by the patients, even those not associated with the most frequent localizations of the disease. In South America, especially in Brazil, prostatic PCM is an important differential diagnosis to prostatic adenocarcinoma and can evolve into a serious symptomatic condition if left untreated.

\section{REFERENCES}

Begliomini H, Gorga CFA, França LCM 1993. Paracoccidioidomicose prostática. Relato de caso e revisão da literatura. J Bras Urol 19: 285-288.

Brito RR, Caprini N 1959. Blastomicose da próstata. Rev Paul Med 54: $116-122$.

Ciconelli AJ, Martins ACP, Costa RB, Fioranai SAS 1969. Blastomicose Prostática. Rev Bras Cirurg 57: 208-11.
Crawford ED 2003. Epidemiology of prostate cancer. Urology 62: 3-12.

Dhundee J, Maciver AG 1991. An immunohistological study of granulomatous prostatitis. Histopathology 18: 435-441.

Eickenberg H-U, Amin M, Lich R Jr 1975. Blastomycosis of the genitourinary tract. J Urol 113: 650-652.

Faber ER, Leahy MS, Meadows TR 1968. Endometrial blastomycosis acquired by sexual contact. Obst Gynec 32: 195-199.

Kalmar EM, Alencar FE, Alves FP, Pang LW, Del Negro GM, Camargo ZP, Shikanai-Yasuda MA 2004. Paracoccidioidomycosis: an epidemiologic survey in a pediatric population from the Brazilian Amazon using skin tests. Am J Trop Med Hyg 71: 82-86.

Melo CR, Melo IS, Cerski CT 1992. Leukaemic infiltration, paracoccidioidomycosis and nodular hyperplasia of the prostate. Br J Urol 70: 329-330.

Paniago AM, Aguiar JI, Aguiar ES, da Cunha RV, Pereira GR, Londero AT, Wanke B 2003. Paracoccidioidomycosis: a clinical and epidemiological study of 422 cases observed in Mato Grosso do Sul. Rev Soc Bras Med Trop 36: 455-459.

Prado FL, Prado R, Gontijo CC, Freitas RM, Pereira MC, Paula IB, Pedroso ER 2005. Lymphoabdominal paracoccidioidomycosis simulating primary neoplasia of the biliary tract. Mycopathologia 160: 25-28.

Reis BS, Bozzi A, Prado FL, Pereira MC, Ferreira FE, Godoy P, Moro L, Pedroso EP, Leite MF, Goes AM 2005. Membrane and extracellular antigens of Paracoccidioides brasiliensis (Mexo): identification of a $28-\mathrm{kDa}$ protein suitable for immunodiagnosis of paracoccidioidomycosis. J Immunol Methods 307: 118-126.

Salfelder K, Doehnert G, Doehnert HR 1969. Paracoccidioidomycosis. Anatomic study with complete autopsies. Virchows Arch A Pathol Pathol Anat 348: 51-76.

Severo LC, Kauer CL, Oliveira F, Rigatti RA, Hartmann AA, Londero AT 2000. Paracoccidioidomycosis of the male genital tract. Report of eleven cases and a review of Brazilian literature. Rev Inst Med Trop Sao Paulo 42: 38-40.

Stewart BG 1964. Epididymitis and prostatitis due to coccidioidomycosis: a case report with 5-year followup. J Urol 91: 280-281.

Stillwell TJ, Engen DE, Farrow GM 1987. The clinical spectrum of granulomatous prostatitis: a report of 200 cases. J Urol 138: 320-323.

Wilt TJ, Brawer MK 1999. Clinical evidence: non-metastatic prostate cancer. West J Med 171: 97-101. 